\title{
Enhancing Robot Teleoperator Situation Awareness and Performance using Vibro-Tactile and Graphical Feedback
}

\author{
Paulo G. de Barros, Robert W. Lindeman, and Matthew O. Ward
}

Worcester Polytechnic Institute

\begin{abstract}
Most of the feedback received by operators of current robotteleoperation systems is graphical. When a large variety of robot data needs to be displayed however, this may lead to operator overload. The research presented in this paper focuses on offloading part of the feedback to other human senses, specifically to the sense of touch, to reduce the load due to the interface, and as a consequence, to increase the level of operator situation awareness. Graphical and vibro-tactile versions of feedback delivery for collision interfaces were evaluated in a search task using a virtual teleoperated robot. Parameters measured included task time, number of collisions between the robot and the environment, number of objects found and the quality of post-experiment reports through the use of sketch maps. Our results indicate that the combined use of both graphical and vibro-tactile feedback interfaces led to an increase in the quality of sketch maps, a possible indication of increased levels of operator situation awareness, but also a slight decrease in the number of robot collisions.
\end{abstract}

KEYWORDS: virtual reality, robot teleoperation, multi-sensory interfaces, vibro-tactile feedback, collision proximity detection.

IndeX Terms: H.5.2 [User Interfaces]: Haptic I/O, Evaluation/methodology; H.5.1 [Multimedia Information Systems]: Artificial, augmented and virtual realities; I.2.9 [Robotics]: Operator interfaces.

\section{INTRODUCTION}

The process of robot teleoperation may be divided into four primary activities: sensing the state of the robot and the remote environment, making sense of such state, deciding on the next action to be taken, and carrying out that action. Any of these steps may make use of automation. The human-robot interaction (HRI) cycle in Figure 1 happens indefinitely as the task is carried out.

In the case of urban search-and-rescue (USAR), the main focus area of this paper, little automation is generally present, though the use of point navigation has become a common approach in robot teleoperation [22][17][29][30].

USAR teleoperation is generally done through the use of ordinary input devices such as keyboard, mouse, and joystick. Most if not all of the information sensed from the robot is presented in a graphic display. During a mission, the operator uses this interface not only as a means to understand the state of the robot and its surrounding environment, but also as a tool to complete mission goals.

WPI CS Dept., 100 Institute Road, Worcester, MA, USA, 01609.

$\{$ pgb, gogo,matt $\} @$ cs.wpi.edu

IEEE Symposium on 3D User Interfaces 2011

19-20 March, Singapore

978-1-4577-0064-4/11/\$26.00 @2011 IEEE

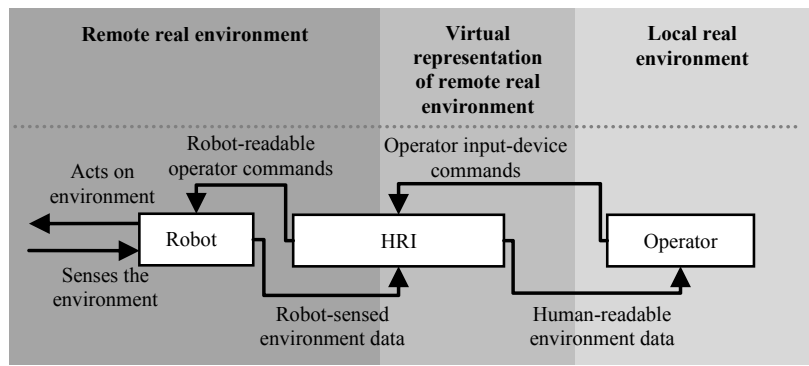

Figure 1. Simple representation of interaction cycle between robot and operator in an HRI system.

Depending on how data is represented on screen, succeeding in both of these tasks may turn out to be very cognitively demanding. This increase in cognitive load may cause a decrease in operator situation awareness (SA) [12], and hence hinder the performance of the entire HRI system [10][24][29].

The research presented here aims at evaluating the impact on SA and performance when part of the data transmitted by the robot is displayed to the operator using senses other than vision. Specifically, the proposed interface uses a body-worn vibro-tactile display to provide feedback to the operator for collision proximity between the robot and the remote environment. In a four-way comparison, the use of vibro-tactile feedback is compared with the use of no feedback, the use of graphical feedback, and the use of both types of feedback in the performance of a simple search task.

Results indicate that the use both types of feedback led to an improvement in the quality of sketch maps, a potential indication of increase in the operator level of situation awareness. A slight improvement in collision avoidance was also detected.

The remainder of this paper is organized as follows. Section 2 summarizes the current work on HRI and vibro-tactile interfaces. Section 3 presents the interface studied. Section 4 describes our experiment hypotheses. The user study methodology is presented in section 5. Section 6 contains the user study results. The analysis is done in section 7. Conclusions are presented in section 8 .

\section{Related Work}

Robot interfaces for USAR systems have been proposed and tested for more than a decade [23]. Nevertheless, a standard model or set of guidelines for HRI has not yet been widely accepted.

Drury [10] has pointed out a set of preliminary guidelines for HRI interfaces. Scholtz [27] has specified a set of general recommendations for designing intelligent systems that may also be applied to HRI interfaces. Common metrics for HRI have also been discussed [28]. In addition, higher-level interfaces for robot socialization and learning have been a point of debate and research in the community [1][22]. Moreover, the Association for the Advancement of Artificial Intelligence (AAAI) Rescue Robotics Competition has been raising and identifying interesting issues about HRI interface design [29]. 
We have analyzed the interface features currently present in different interface designs from a variety of research projects [10][11][13][24][25][29][30] (see [9] for details). It appears that the focus of most of these interface designs is on improving situation awareness and efficiency, but not on reducing cognitive load and creating systems that are scalable, portable, reusable or robust. The interface proposed here aims to change this HRI interface development trend by attacking the problem of the operator's cognitive load through the use of feedback devices in addition to graphical ones.

Tactile feedback has been used in Virtual Reality (VR) as an experimental interface and an outlet for cognitive overload [5]. In addition, the VR, Human-Computer Interaction (HCI) and 3D User Interaction (3DUI) research communities have been studying different modalities of vibro-tactile and multi-sensorial cues for providing collision [3][4][26] and other types of feedback [6][14] for different tasks. Some strategies for handling collision avoidance smoothly have also been proposed [15].

As summarized by Lindeman [18][19][20] and Zelek \& Asmar [31], tactile cues have been used as display devices on different parts of the body. Nevertheless, when designing a haptic interface, it is important to consider the fact that human tactual perception sensitivity varies according to body location. Sensitivity on the fingers, lips, and tongue is much higher than on the back and shins, for example, and may affect the user's SA of the remote environment. Other areas of research have also benefited from this type of feedback, such as Cassineli's [8] employment of vibrotactile feedback as an object-collision avoidance mechanism for blind people.

Lindeman provided a classification scheme of the types of contact that can be potentially represented by vibro-tactile displays in virtual environments (VEs). For tele-operated robots, this classification is applicable without further modification if we replace the virtual environment with the real remote one.

Although we focus on vibro-tactile feedback in HRI, other input and output (I/O) devices could also be more extensively used in HRI, such as spatial audio, low-resolution stereoscopic displays, and motion capture. They should be adapted to current HRI systems by leveraging current VR I/O techniques [5].

\section{Collision-Proximity Feedback INTERface}

We have designed a Collision-Proximity Feedback (CPF) interface that attempts to follow a superset of the guidelines proposed in the field and merge successful features from interface designs tested by other research groups.

In this work, we use a simulated robot instead of a physical one, in order to quickly prototype different interface elements. Our design (Figure 2) uses as a starting point the interface proposed by Nielsen [24][25]. The operator is presented with a third-person view of a $3 \mathrm{D}$ virtual representation of the robot, called its avatar. The real robot and its avatar have the approximate size of a standard search robot $(0.51 \mathrm{~m} \times 0.46 \mathrm{~m} \times 0.25 \mathrm{~m})$. Data collected by the robot sensors is also presented, including a video feed from a pan-tilt camera mounted on the robot, location of object surfaces near the robot, and potential collision locations. The robot camera has a field-of-view of $60^{\circ}$. A panel located in front of the robot avatar projects data from the robot's simulated camera. The camera, and hence the panel, can be rotated about both the vertical and horizontal axes, up to an angle of $\pm 100^{\circ}$ horizontally and $\pm 45^{\circ}$ vertically, relative to the front of the robot. The camera-panel rotations occur relative to the robot avatar and match the remote virtual robot camera rotations controlled by operator input.

A ring surrounding the robot avatar indicates imminent collisions near the robot, similar to the Sensory EgoSphere proposed by Johnson [17] but with a more specific purpose; the brighter the red color in the ring the closer to a collision point the robot is. The ring was not made continuous to make more explicit the different feedback directions and to reduce the amount of occlusion it caused. The ring is not transparent to avoid interference from the surrounding virtual objects' colors on the feedback color provided by the ring. Its radius and height were set so that it could be seen in its entirety from the back, it would not occlude the front of the robot and it was aligned with the height of the robot sensors.

The same type of feedback is also provided as vibration through the vibro-tactile interface, henceforth called the TactaBelt [18]. The TactaBelt consists of eight pager motors, also called tactors, arranged in a ring around the robot operator's torso, with the motors spaced evenly. The more intense a tactor in the TactaBelt vibrates, the closer the robot is to colliding in that direction, similar to the feedback technique proposed by Cassineli [8].

Both visual and vibro-tactile feedback interfaces are only activated when an object is within a distance $d$ from the robot $(d \leq$ $1.25 \mathrm{~m})$. Directional feedback values for the ring-cylinder redness and tactor vibration vary continuously from near zero, when distance is close to $d$, to near their maximum values when the robot is about to collide with the object.

A map of the environment is gradually projected on the ground in the form of blue lines as the robot captures data from the environment. These blue lines represent the locations of objects and wall surfaces detected by the robot sensors. The robot avatar position on the map matches the virtual robot position in the real world VE. These positions are always synchronized.

Figure 2 illustrates the main components of the graphical interface. In this illustration, the robot passed close to a large number of the objects in the scene before and thus most of the walls in the environment can be seen on the blue map. The eight cylindrical components of the graphical ring are presented in different levels of red saturation around the robot avatar, in front of which the camera panel is seen. A timer is presented in the top right hand corner of the screen. It is triggered once the training session finishes and the robot is transferred to another VE where the actual experiment takes place. This transition and both VEs are further described in section 5.1 .

The controller used in the experiment was a Sony PlayStation2 Dual-shock (Figure 3a). The controller allowed the subject to move the robot backward and forward and rotate the robot to the left or right. The robot rotation was assumed to be controlled using differential drive, which meant the robot rotation could happen in place or while in movement. The pan-tilt movement of the camera was inverted and moving the joystick forward would move the camera down. This camera control option was chosen based on subject preference during a pilot study.

The TactaBelt was custom made, and consists of a set of eight tactors located at the cardinal and intermediate compass points, with forward being North (Figure 3b). Each tactor (Tokyo Parts Industrial Co., Ltd., Model No. FM37E, 2.5-3.8V at 40mA, see [19] for more details) is a simple DC motor with an eccentric mass, similar to those used in mobile phones. Individual PulseWidth Modulation (PWM) signals produced by the TactaBox system control the level of vibration for each tactor, and are manipulated through the interface API of the TactaBox [18].

The machine used for running the experiment was a Dell XPS 600 with 2 GB RAM and a Pentium (R) D Dual-core $3 \mathrm{GHz}$ processor. The environment was run in a window with resolution of 1280x1024 at an average frame rate of 30 frames-per-second (fps) on an LCD monitor (Viewsonic Q20wb) placed on top of an office table and approximately aligned with the subject's view height. The monitor was positioned at an approximate distance of $0.5 \mathrm{~m}$ from the subject eyes. The graphics card used was a GeForce 7800 GTX with 256MB of memory. 


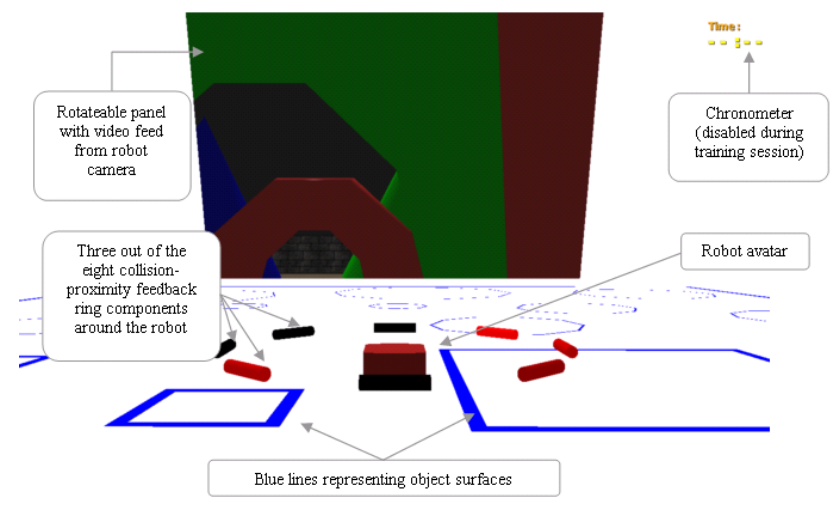

Figure 2. Main Components of the graphical interface.

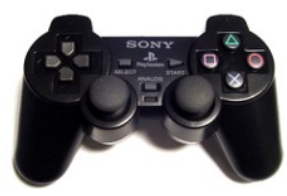

(a)

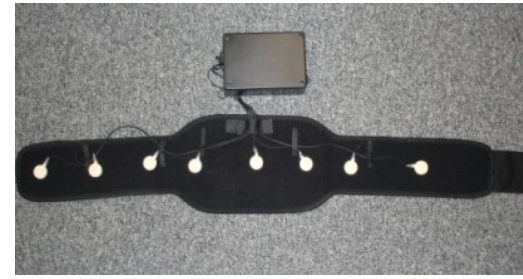

(b)

Figure 3. Interface used in addition to the standard LCD monitor: (a) PlayStation 2 dual-shock controller; (b) TactaBelt.

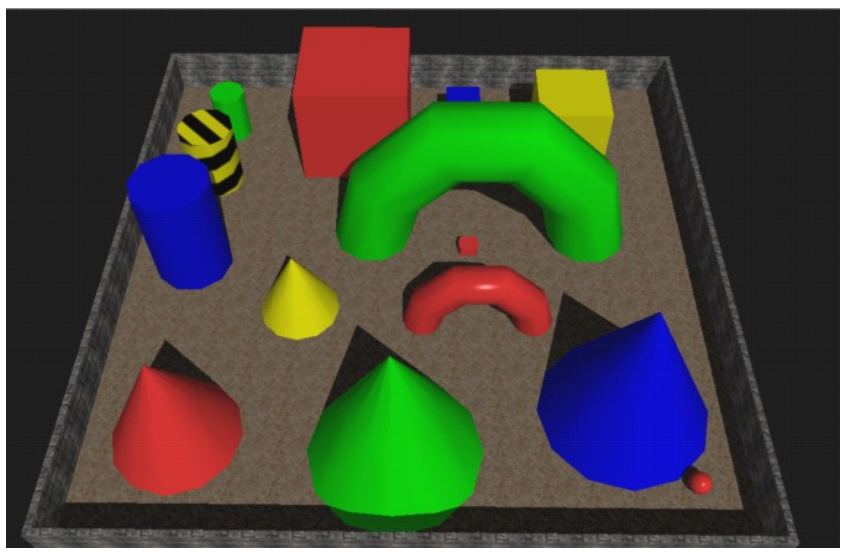

Figure 4. Training environment from a bird's eye view.

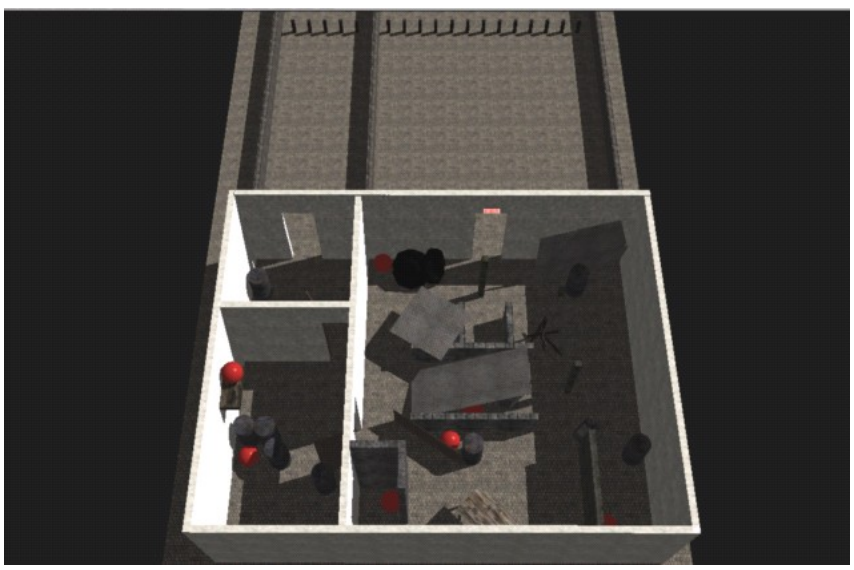

Figure 5. VE for the experiment from a bird's eye view.

\section{4}

Previous results obtained from other research groups have shown improvement in performance when using vibro-tactile displays $[3][4][6][14][19][26]$ and enhanced interfaces [17]. Based on these results, we claim that the use of the graphical ring and the TactaBelt should cause an improvement in subjects' perception of the surrounding environment, indicating an increase in their situation awareness level. This should be especially evident through a reduction in the number of collisions. Improvement should also be visible in the results collected by other dependent variables. By making navigation more intuitive with the addition of directional feedback, and less visual with the addition of vibrotactile feedback, subjects using the enhanced interfaces will be able to focus more on the task, find a larger number of objects, and better understand how the environment is organized. The first two ability-enhancement effects may be seen as a consequence of a lower cognitive load while the second and third may be seen as a result of higher levels of situation awareness. Therefore, task time, number of collisions, number of objects found, and understanding of the positions of objects are measurements that are relevant to the validation or rejection of our hypothesis. This hypothesis is split into the following hypotheses.

Our first hypothesis (H1) is that subjects using either the vibro-tactile or the graphical ring feedback interface should have an increase in navigational performance and situation awareness (SA) measured by four factors: a reduction in the number of collisions (local SA improvement), a reduction in the time taken to perform the task (performance improvement), an increase in the number of objects found (performance improvement) and a better reporting of the location of the objects and understanding of the environment (global SA improvement) in relation to the control group, which is using neither interface. The first two factors are objective experimental measures. The third and fourth factors are obtained using the post-test map sketches, henceforth called sketch maps.

Our second hypothesis (H2) is that subjects who are using both the vibro-tactile and the graphical ring feedback interfaces should have an even larger increase in navigational performance and situation awareness measured by the same four factors compared to subjects using either or none of these.

\section{EmPIRICAL STUdY}

A user study was carried out to confirm our hypotheses that the use of either or both feedback modalities would result in an improvement in operator performance and situation awareness. There are at least two ways to compare user interfaces. The first (lab interfaces) attempts to hold constant all aspects of the interfaces being compared, with the exception of the independent variables. These experiments allow statements to be made about the effects of the variations in the interfaces, but suffer from the fact that in the field, an interface designer might construct a vastly different interface given the value of the independent variable. This leads her to compare interfaces that vary greatly, but are more "optimized" given the independent variable, producing a second type of experiment, where interfaces are constructed that represent the best efforts of the UI designer given the independent variables being studied (fielded interfaces).

For our study, we opted for a fielded interface experiment. We designed our interface to approximate an interface that is actually used by research groups and experts in performing USAR tasks as much as possible. This was done by adding to our interface common features of these interfaces, such as a map of the world and allowing the subject to navigate the robot and perform the search task. Despite the challenges in having many potential 
variables that may affect subject performance, it was only by taking this approach that we could detect the correct effect of inserting a multi-sensorial proximity feedback interface to the application in a reasonably realistic USAR context.

\subsection{Methodology}

The task subjects had to complete consisted of locating red spheres, with a radius of $0.25 \mathrm{~m}$, in the ruins of a small closed environment. Subjects were asked to search for as many red spheres as they could while avoiding collisions between the robot and the environment. They were also asked to perform the task in as little time as possible. A total of nine spheres were hidden. Subjects did not know in advance the number of spheres, and after the experiment was over, they were asked to sketch a map of the task space with the approximate location of the spheres.

The user study consisted of a between-subjects experiment. The independent variable was the type of CPF interface. Subjects were divided into four groups: the first group ("None") operated the robot without using any CPF interface. The second ("Ring") received feedback from the graphical ring. The third ("Vibrotactile") received feedback from the TactaBelt. The fourth ("Both") received feedback from both the graphical ring and TactaBelt. All subjects wore the TactaBelt, but the interface was not active during the experiment for most of them. The neoprene belt with Velcro-attached tactors adapted to most subjects waists without problems. When subjects were very slim or the opposite, the tactors were repositioned so they were correctly aligned with the cardinal and intermediate directions relative to the subject's waist. Subjects could control the robot and its camera using the two analog joysticks of the gamepad. Two trigger buttons on the gamepad allowed subjects to take pictures of the environment. These pictures were used by subjects in the map-sketching exercise that followed the search task as further explained below.

The dependent variables were the number of collisions, the time taken to accomplish the search task, the number of spheres found, and the quality of the sketch maps.

The user study can be summarized by a list of eight steps for each subject, some of which are further explained in the paragraphs following this list.

1. Institutional Review Board (IRB) approved consent forms were read and signed;

2. Demographic information was collected;

3. The experiment instructions and a Q\&A session occurred;

4. Robot controls for the experiment were explained;

5. The training session task was explained, questions answered, and the subject started this session when ready;

6. After the training session, the experimenter explained that the robot would be moved to the world where the real task would be performed and briefly reviewed the objective of the latter. The experiment started when the subject was ready;

7. During the main experiment, the experimenter took general notes about the subject and his performance;

8. Once the main experiment task was over, the subject filled in a post-task questionnaire containing the sketch map and asking for general experiment feedback.

The demographics questionnaire collected subject information about their gender, age, how often they played video games and used or worked with robots. For the last two questions, the possible answers were one of the four following Likert scale values: "daily" (1), "weekly" (2), "seldom" (3) or "never" (4). Other than the answer from these questions, no general spatial ability information was collected from subjects.
A single page of instructions contained a description of the experiment, the task to be completed, the interface, and how subjects should behave before, during, and after the experiment.

The training session happened in a virtual training room $(15 \mathrm{~m} \mathrm{x}$ $15 \mathrm{~m})$ larger than the one for the real task session $(8 \mathrm{~m} \times 10 \mathrm{~m})$. The training room (Figure 4) contained large colored geometric primitives. A single red sphere was hidden behind one of these primitives. The training task for this room was to find the hidden red sphere and take a picture of it. This gave the subject time $(\sim 4$ min.) to practice and become accustomed to the robot controls. During this session, if subjects seemed to be already comfortable with the robot controls but were having problems in finding the red sphere, the experimenter would intervene and give them hints on the location of the sphere so that they could practice taking pictures, ask questions, and then move on to the real experiment.

In the real task room, objects such as doorways, barrels and tables where represented in their size in reality. The data on the location and time of the collisions were recorded as well as the time spent in performing the task. Additionally, the periods of time spent during the training session and sketching the location of the spheres were recorded for some of the subjects; the idea of collecting such data only came up half-way through the subjects.

The post-task questionnaire asked subjects to report the number of spheres found and their location by sketching a map of the environment. They were provided with the pictures they took during their traversal of the environment to help them in sketching. The images were displayed with a resolution of $800 \times 640$ pixels on a Web page.

The sketch maps were evaluated following the criteria proposed by Billinghurst \& Weghorst [2]. The first criterion was map goodness, which was evaluated on a scale from 1 to 5 , instead of the original scale from 1 to 3 . The criterion for grading map goodness was how well the sketched map would help in guiding someone through the environment. The second criterion was counting the number of objects of different classes or groups that were drawn. The objects were divided into three groups: walls, doorways, and debris. These groups were scored separately. Each object found corresponded to a one-point increment to their object group score. The third criterion was a general scoring and analysis of the correct placement of objects relative to other nearby objects. Sphere placement was not considered during grading of any criteria, since the pictures taken would allow subjects to position them correctly relative to nearby objects most of the time.

\subsection{Virtual Environment}

The robot side of the system was simulated using a VE. In fact, two VEs, built using the C4 game engine (www.terathon.com), were used in our application. The first world was the simulated world where the robot was present and where it should complete the search task (Figure 5). In the context of the AAAI Rescue Robotics Competition, the environment is qualified as being the level yellow of the competition, where the robot traverses the entire world by moving around the same ground level with some debris spread across the floor [16]. The second world represented the robot teleoperation interface as seen from the operator's point of view (Figure 2).

\subsection{Other Materials}

Other material used in this user study, such as the script used by the experimenter, the information contained in the user study instruction sheet, and the questions contained in the user study post-task questionnaire can be provided by contacting the authors. 


\section{Results}

All the comparisons among the results presented in this section were made using a single-factor ANOVA with confidence level of $\alpha=0.05$. The $f$ and $p$ vlaues for the data analyses that resulted in relevant and statistically signifcant results are presented on the tables in this section. Further details about results that were not statistically signifcant can be obtained upon authors request.

\subsection{Demographic Information}

A total of 13 female and 14 male university students participated in the user study. All groups had 7 subjects, except group 2 ("Ring"), which had 6. A comparison between genders for the dependent variables showed no statistically significant difference (SSD). The mean age was 20.52, with standard deviation of 5.24.

No SSD was found among groups in terms of videogame experience, although subjects in group "Both" had a lower average than others, that is, they had a slightly higher level of experience. Interestingly, videogame experience did prove to have a statistically significant effect on the result for number of collisions between groups "Weekly" and "Never" $(f=5.18$, $p=0.04)$. Groups with different levels of videogame experience were also compared in terms of task time, number of spheres found, and map goodness, but none of these showed any SSDs.

Only two groups had subjects with robot experience. However, robot experience did not have any statistically significant effect on the results of any of the dependent variables.

\subsection{Sketch Maps}

Map samples sketched during the experiment as well as the blueprint of the original scene are presented in Figure 6.

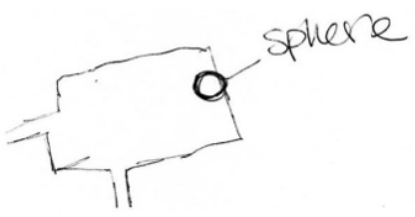

(a)

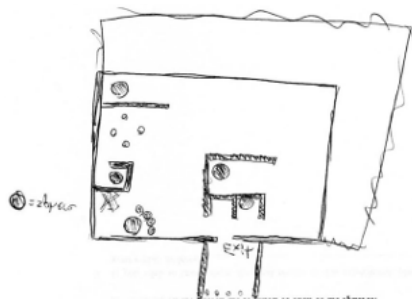

(c)

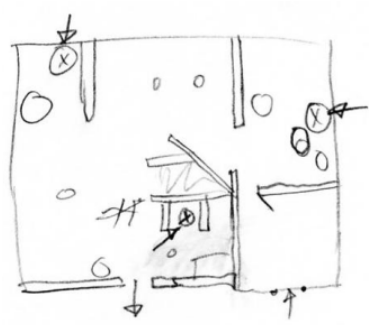

(e)

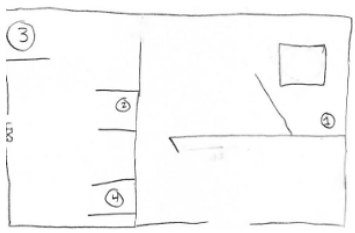

(b)

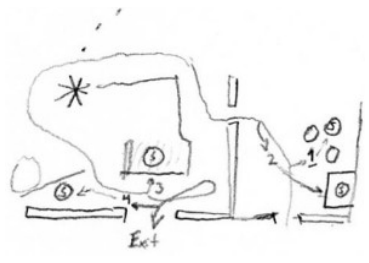

(d)

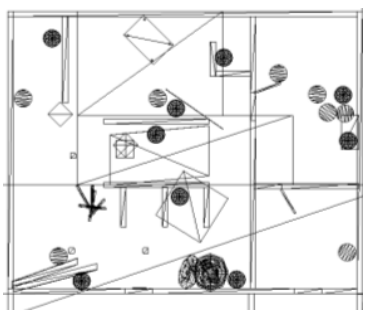

(f)
Figure 6. Sketch map samples for different grades: (a) goodness score $=1$; (b) goodness score $=2$; $(\mathrm{c})$ goodness score $=3$; $(\mathrm{d})$ goodness score $=4$; $(e)$ goodness score $=5$; (f) original map.
Maps scored as 1 provided no help as a guidance tool through the environment. Maps scored as 2 had the description of a few features of the environment represented it with a large number of mistakes in terms of spatial representation. Maps scored as 3 had some features of the environment well placed and described in text, but still had major errors in their sketches, such as the number of rooms and doorways. Maps scored as 4 described the environment correctly except for the misplacement of some objects and walls. Maps graded as 5 had the environment almost completely correct and all the objects found were correctly placed.

Some subjects also added extra features to their descriptions of the scene, by drawing the approximate path they went through during the search task (Figure 6d) or the order with which they found the spheres and how these related to the pictures taken (Figure $6 \mathrm{~b}$ and Figure $6 \mathrm{c}$ ). Almost half of the subjects failed to make good representations of the environment, and had their maps graded as 1 or 2 . When comparing groups with different levels of map goodness to task time, no SSD was found. Good and poor maps were sketched by subjects who spent from 4 minutes to 20 minutes in the environment.

Since sketch maps must be scored by a person, results may be affected by subjectivity and thus scoring effectiveness needs to be validated. In this study, the first evaluation criterion, map goodness, was used as a general score for map quality. However, we ensured map quality results were in accordance with the results obtained by the other more specific criteria: object counts for walls, doorways and debris, and their relative position to nearby objects. Once again, notice that the location of spheres was not among the map goodness evaluation criteria. All the maps were graded for all the criteria by the same person. Please refer to [9] for more details.

The standard mean $(\bar{x})$, standard deviation $\left(s_{n}\right)$ and median $(\tilde{x})$ results for these map evaluation criteria are presented in Table 4.

Table 1. Summary of sketch map results.

\begin{tabular}{lccc}
\hline Variable & $\bar{x}$ & $S_{n}$ & $\tilde{x}$ \\
\hline Number of walls sketched & 15.33 & 5.23 & 16 \\
Number of doorways sketched & 3.74 & 1.20 & 4 \\
Number of debris sketched & 5.7 & 4.44 & 5 \\
Number of correct positioning of objects & 2.81 & 2.15 & 2 \\
\hline
\end{tabular}

\subsection{Core Results}

Multimodality was detected in the histograms for number of collisions, task time, and number of spheres found. In order to normalize these results in terms of time and reduce the effect of multimodality, we have also adopted in our analysis the measures of number of spheres found per minute and number of collisions per minute instead of considering only the independent measures of number of spheres found, number of collisions, and task time.

\subsubsection{Evaluation of the Reduction in the Number of Robot Collisions}

For the number of collisions per minute, no statistically significant difference was found amongst groups, although a visually perceptible difference in results is noticeable among groups (Figure 7), where the "Both" group has the lower result. Due to no $\mathrm{SSD}$, the part of both hypotheses referring to an improvement in the number of collisions caused by the use of CPF interfaces is rejected.

A comparison of the number of collisions between groups showed SSDs between groups ("None", "Ring") and ("Ring", "Vibro-tactile") as presented in Table 2. Again, no difference was found for any of the other pairs of groups. For group "Both", the cause for non-significant difference in the results might have been the high variation found in subject data from this group $\left(s_{n}\right.$ : 
33.30), although the largest variation value was obtained in group "Ring". However, the p-value for the ("Ring", "Both") pair is 0.06. This is close to being significant. We conjecture that this indicates how the redundant feedback provided by vibro-tactile interface seems to balance out negative effects on collision avoidance due to occlusion caused by the graphical ring interface, and hence reduces the average number of collisions ( $\bar{x}: 27.28)$.

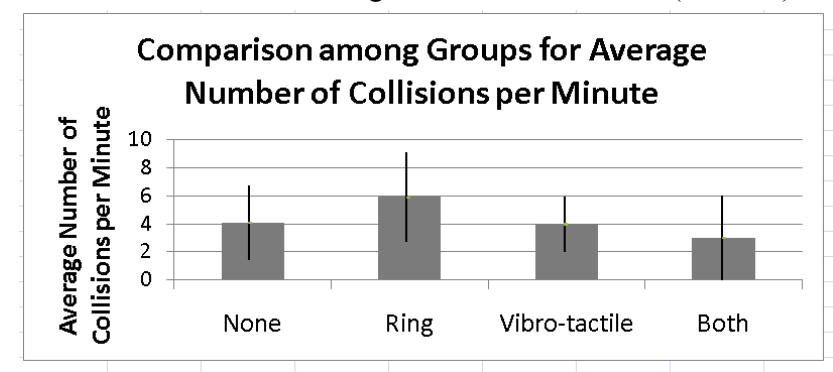

Figure 7. Comparison among interface groups for number of collisions per minute (average \pm 1 std. deviation)

Table 2. Comparison of number of collisions among groups using different CPF interfaces.

\begin{tabular}{|c|c|c|c|}
\hline & $\begin{array}{c}\text { Ring } \\
(\bar{x}: 71.67 \\
\left.s_{n}: 48.34\right)\end{array}$ & $\begin{array}{l}\begin{array}{c}\text { Vibro- } \\
\text { tactile }\end{array} \\
(\bar{x}: 29.28 \\
\left.s_{n}: 2.80\right)\end{array}$ & $\begin{array}{c}\text { Both } \\
(\bar{x}: 27.28 \\
\left.s_{n}: 3.30\right)\end{array}$ \\
\hline $\begin{array}{l}\text { None } \\
\left(\bar{x}: 20.43 s_{n}: 5.59\right)\end{array}$ & $\begin{aligned} f & =6.69 \\
p & =0.025\end{aligned}$ & $\begin{array}{l}\mathrm{f}=1.05 \\
\mathrm{p}=0.32\end{array}$ & $\begin{array}{l}f=0.27 \\
p=0.61\end{array}$ \\
\hline $\begin{array}{l}\text { Ring } \\
\left(\bar{x}: 71.67 s_{n}: 48.34\right)\end{array}$ & & $\begin{array}{lr}f & =5.08 \\
p & .04\end{array}$ & $\begin{array}{l}f=4.17 \\
p=0.06\end{array}$ \\
\hline $\begin{array}{l}\text { Vibro-tactile } \\
\left(\bar{x}: 29.28 s_{n}: 12.80\right)\end{array}$ & & & $\begin{array}{l}f=0.03 \\
p=0.87\end{array}$ \\
\hline
\end{tabular}

\subsubsection{Evaluation of the Increase in Number of Objects Found}

For the number of spheres found per minute (Figure 8), a statistically significant difference between groups "Ring" and "Both" was found ( $f=11.17, p=0.0066)$. This only indicates that the use of the Ring interface by itself seems to lead to a smaller number of spheres found while the vibro-tactile interface seem to have no effect on improving the number of spheres found. This means that the part of both hypotheses that refers to an improvement in the number of spheres found caused by the use of $\mathrm{CPF}$ interfaces is also rejected. The fact that "None" has the highest mean indicates that the use of feedback interfaces has some impact on subjects' cognitive load and search performance, but such impact is not statistically significant.

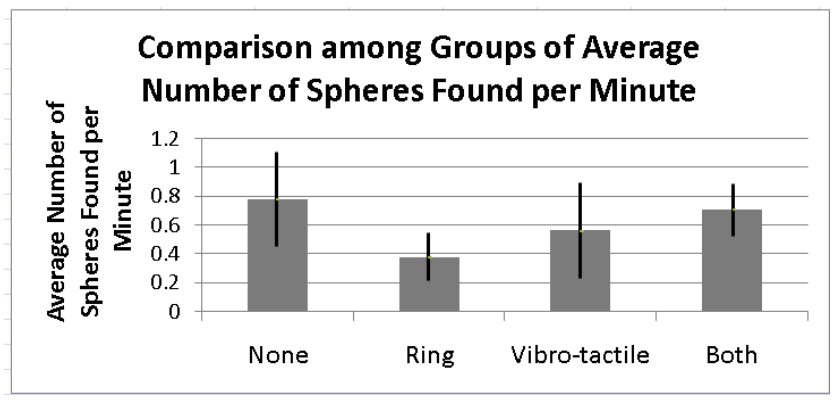

Figure 8. Comparison among interface groups number of spheres found per minute (average \pm 1 std. deviation).

A comparison of the number of spheres found among interface groups also showed no SSD. Nevertheless, a slight increase is perceived in the median value of the number of spheres found as the interface group changes from group "None" (no interface enhancement is used) moving through groups "Ring" and "Vibrotactile" (some interface enhancement is used) towards group "Both" (both interface enhancements are used), the latter having the highest median value (Figure 9).

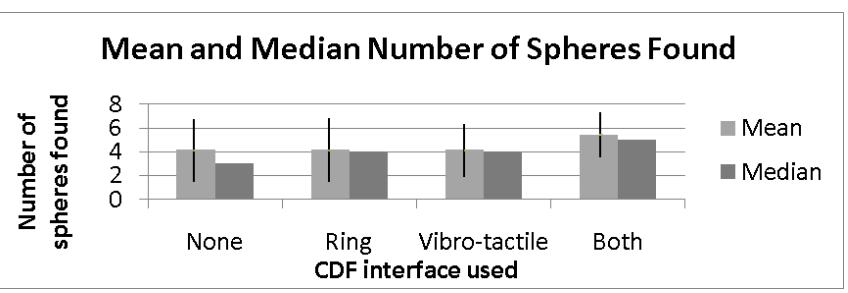

Figure 9. Means ( \pm 1 std. deviation) and medians of the number of spheres found per group.

\subsubsection{Evaluation of the Improvement in Environment Understanding}

When comparing map goodness with the type of CPF interface used, a SSD was found only between groups "None" and "Both" (Table 3). Figure 10 more clearly represents this variation for group "Both". Notice that there is a trend towards significance between groups "Vibro-tactile" and "Both". This might be a consequence of using the "Ring" interface by group "Both".

Table 3. Comparison of map goodness among groups using different CPF interfaces.

\begin{tabular}{|c|c|c|c|}
\hline & $\begin{array}{c}\text { Ring } \\
(\bar{x}: 2.5 \\
\left.s_{n}: 1.64\right)\end{array}$ & $\begin{array}{c}\begin{array}{c}\text { Vibro- } \\
\text { tactile }\end{array} \\
(\bar{x}: 2.43 \\
\left.s_{n}: 1.27\right)\end{array}$ & $\begin{array}{c}\text { Both } \\
(\bar{x}: 3.57 \\
\left.s_{n}: 0.79\right)\end{array}$ \\
\hline $\begin{array}{c}\text { None } \\
\left(\bar{x}: 2.57 s_{n}: \mathbf{0 . 7 9}\right)\end{array}$ & $\begin{array}{l}f=0.0 .1 \\
p=0.92\end{array}$ & $\begin{array}{l}f=0.06 \\
p=0.80\end{array}$ & $\begin{array}{l}\mathbf{f}=\mathbf{5 . 6 5} \\
p=0.03\end{array}$ \\
\hline $\begin{array}{c}\text { Ring } \\
\left(\bar{x}: 2.5 s_{n}: 1.64\right)\end{array}$ & & $\begin{array}{c}f=0.008 \\
p=0.93\end{array}$ & $\begin{array}{l}f=2.37 \\
p=0.15\end{array}$ \\
\hline
\end{tabular}

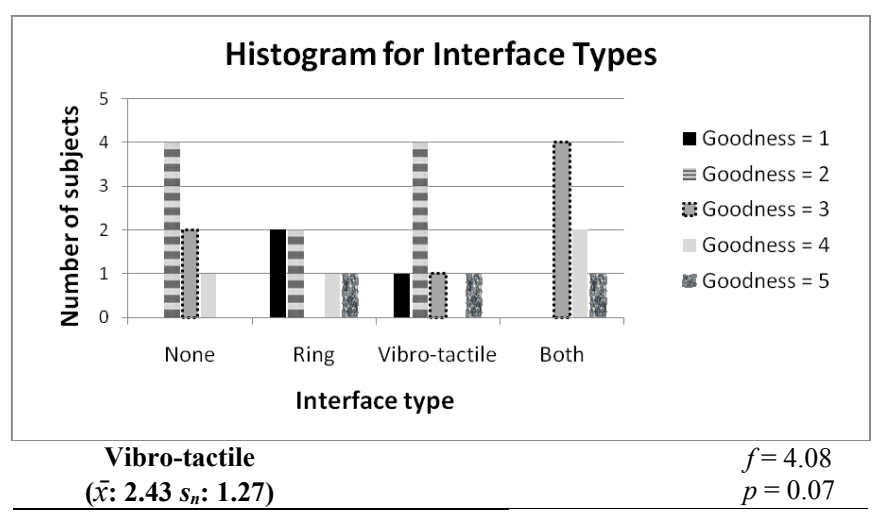

Figure 10.

Histogram for interface types colored according to levels of map goodness.

Notice in the group "Both" graph column the absence of sketch maps rated with goodness levels 1 or 2. This is an important result, because it may indicate the positive effect caused by the $\mathrm{CPF}$ interfaces on subject's SA levels. In addition, notice a larger variation in map goodness for groups "Ring" and "Vibro-tactile" compared to group "None". It indicates that using CPF interfaces separately may result in a positive or negative effect on individual operators, but no improvement on average. 


\subsubsection{Evaluation of the Reduction of Task Time}

A comparison of task time among CPF interface groups led to no $\mathrm{SSD}$, that is, these interfaces have little to no impact on task time.

\subsubsection{Map Goodness, Number of Objects Found and Number of Collisions Relationship}

Last, correlations deemed relevant are presented in Figure 11. For these, correlation coefficients $R$ were considered as insignificant if $|R|<0.1$, weak if $0.1 \leq|R|<0.3$, medium if $0.3 \leq|R|<0.5$ and strong if $0.5 \leq|R| \leq 1.0$.

The first correlation found (Figure 11a) was a medium one between map goodness and the number of spheres found per minute by subjects $(R=0.41)$. The second (Figure $11 \mathrm{~b})$ was a medium negative correlation between map goodness and the number of collisions per minute $(R=-0.44)$.

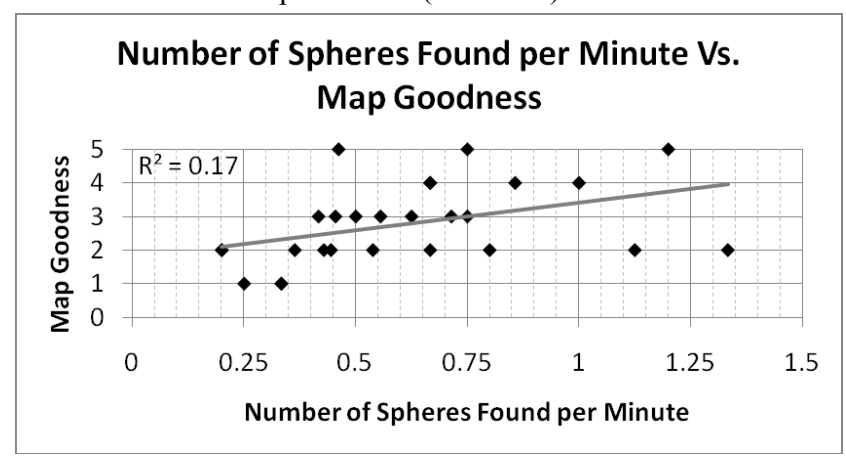

(a)

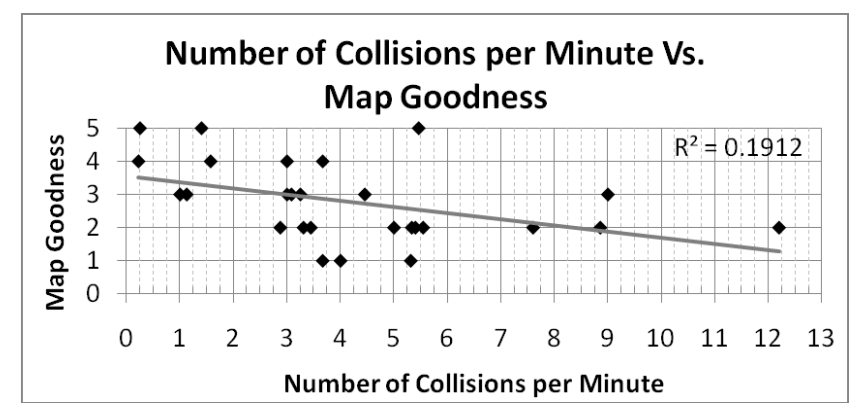

(b)

Figure 11. Map goodness correlation with (a) number of spheres found per minute and (b) number of collisions per minute.

\section{Discussion}

Comments on the results and how they relate to the hypotheses are presented in this section. Gender, age, robot experience, and videogame experience did not have any biasing effect on the results obtained from this user study. However, our analysis confirms that videogame experience may bias results in case the groups are not properly balanced as in our study. This is an important variable to consider in future similar HRI studies.

Our results have shown that the use of CPF interfaces had no negative or positive effect in the task-time and number of spheres found.

With respect to number of collisions, the "Ring" interface group performed worse than interface groups "None" and "Vibrotactile". This might be due to the fact that the ring itself occludes part of the blueprints on the ground around the robot, making it harder for the operator to visually discern closeness of nearby objects. This negative effect seemed to have been counterbalanced by the use of complementary vibro-tactile feedback in the "Both" group, whose collision-count was not statistically worse than any of the other groups and whose average collisioncount per minute had the smallest value. However, the group "None" was the one that generated the lowest average number of collisions. This seems to indicate that we cannot reach any positive or negative conclusions about the effect of CPF interfaces in collision avoidance and improvement of subject's level of local situation awareness. The most interesting result was that group "Both" outperformed the "None" interface group in map goodness scores. This result shows that the combined use of both $\mathrm{CPF}$ interfaces might have been beneficial for the operator in terms of understanding of the virtual environment and location of objects. This result could be associated with an increase in operator global situation awareness. The fact that the coupled CPF interfaces did not affect task time, number of collisions or spheres found, combined with the fact that task-time had no correlation with increase in sketch map quality, seem support the claim that only $\mathrm{CPF}$ interfaces could have caused the increase sketch map quality. The improvement caused by the use of redundant multi-sensorial feedback goes in hand with previous research results in different tasks and applications [6][14]. The small population that participated in this study (6-7 per group), however, does not allow us to reach that conclusion with statistic soundness yet. A user study with a larger population size would be required.

Last, the correlation results show that map goodness, number of spheres found, and number of collisions with the environment seem to be slightly associated with each other. As mentioned before location of spheres was not part of the map goodness evaluation. This relationship might mean that the more spheres subjects can locate, the higher their SA level, or that the higher their SA level, the more spheres will be found, which is to be expected. It also may indicate that the higher their SA level, the less likely the subjects are to collide with the environment or that the lower the number collisions, the higher their level of SA.

\section{CONCLUSION}

The fact that group "Both" drew better maps than all other groups, and that the vibro-tactile interface had no negative impact for all conditions, may indicate that the use of this interface in conjunction with other graphical $\mathrm{CPF}$ interfaces can improve operator situation awareness without detriment to cognitive load. Interestingly, the results seem to point to an increase in global situation awareness instead of local situation awareness.

In terms of collisions, it appears that the current version of the ring feedback interface needs to be improved for it blocks the operator view of the map blueprint. Although the results with the graphic ring interface were opposite to what our hypotheses stated, we believe that a more in-depth study must be performed in order to verify whether this is indeed an invalid approach.

Some subjects complained about the excessive strength of the vibro-tactile feedback. Others have requested an interface to allow adjustment of the distance from an object below which the belt would start providing feedback. These features have already been adjusted in our next user study.

By looking at the results obtained, the authors believe that the use of multi-sensorial interfaces, including vibro-tactile ones, is an important step in the HRI community to significantly improve interface quality.

Other areas of future work include, first and foremost, using a real robot in our experiments so that situations unforeseen in the simulation may be discovered and dealt with. This will help us validate our interface in an environment that better approximates the real-life situation. Utilizing other more commonly used robot simulation platforms, such as USARSim [7] is also being considered. 
Secondly, we plan to study different ways of providing vibrotactile feedback other than varying vibratory intensity, such as providing vibratory patterns. A framework has already been developed and is also being incorporated into our application and next user study.

Other ideas include the creation of an improved version of the graphical feedback interface that may not necessarily be a ring, and adding more feedback mechanisms from the robot to operator that are already commonly used graphically in HRI interfaces, such as $\mathrm{CO}_{2}$ level meters, multiple camera views, and flashlights.

\section{REFERENCES}

[1] Z.Z. Bien, H.-E. Lee Effective learning system techniques for human-robot interaction in service environment. Knowledge-Based Systems, volume 20, number 5, pages 439-456, June 2007.

[2] M. Billinghurst, S. Weghorst, S. The use of sketch maps to measure cognitive maps of virtual environments. Virtual Reality Ann. Int. Symp. (VRAIS '95), page 40, 1995.

[3] K.J. Blom, S. Beckhaus, Virtual Collisison Notification. Proc. of IEEE Symp. on 3D User Interfaces (3DUI), pages 35-38, March 2010.

[4] A. Bloomfield and N. Badler. Collision Awareness Using Vibrotactile Arrays. Proc. of the IEEE Virtual Reality Conf. (VR), pages 163-170, March 2007.

[5] D. Bowman, E. Kruijff, J. LaViola Jr., I. Poupyrev. 3D User Interfaces: Theory and Practice, parts 2 and 3, pages 27-310, Addison-Wesley, Boston, 2005, ISBN 0-201-75867-9.

[6] J. L. Burke, M. S. Prewett, A. A. Gray, L. Yang, F. R. B. Stilson, M. D. Coovert, L. R. Elliot, and E. Redden. Comparing the effects of visual-auditory and visual-tactile feedback on user performance: a meta-analysis. ICMI '06: Proc. of the 8th Int'l Conf. on Multimodal Interfaces, pages 108-117, New York, NY, USA, 2006. ACM.

[7] S. Carpin, M. Lewis, J. Wang, S. Balakirsky, C. Scrapper. USARSim: a robot simulator for research and education. Proc. of the IEEE Conf. on Robotics and Automation, pages 1400-1405, 2007.

[8] A. Cassinelli, C. Reynolds, M. Ishikawa. Augmenting spatial awareness with Haptic Radar. Tenth Int'l Symp. on Wearable Computers (ISWC), Montreux, Switzerland. Short paper (4 pages), October 2006.

[9] P.G. de Barros, R.W. Lindeman, M.O. Ward. A Multi-sensorial HRI Interface for Teleoperated Robots. Technical Report Series, WPICS-TR-09-09, Computer Science Department, Worcester Polytechnic Institute, 2009.

[10] J.L. Drury, D. Hestand, H.A.. Yanco, J. Scholtz. Design Guidelines for Improved Human-Robot Interaction. Extended Abstracts on Human Factors in Computing Systems (CHI '04), page 1540, April 2004.

[11] J.L. Drury, H.A. Yanco, W. Howell, B. Minten, J. Casper. Changing Shape: Improving Situation Awareness for a Polymorphic Robot. Proc. of the 1st ACM SIGCHI/SIGART Conf. on Human-Robot Interaction, pages 72-79, March 2006.

[12] M.R. Endsley, D.G. Garland. Theoretical Underpinning of Situation Awareness: A Critical Review. Situation Awareness Analysis and Measurement, Lawrence Erlbaum, Mahwah, NJ, 2000.

[13] M.A. Goodrich, E.R. Boer, H. Inoue. A Characterization of Dynamic Human Braking Behavior with Implications for ACC Design. Proc. IEEE/IEEJ/JSAI Conf. on Intelligent Transportation Systems, Tokyo, Japan, October 1999.

[14] I. Herbst and J. Stark. Comparing Force Magnitudes by Means of Vibro-Tactile, Auditory and Visual Feedback. IEEE Int'l Workshop on Haptic Audio Visual Environments and their Applications (HAVE), pages 67-71, 2005.

[15] J. Jacobson and M. Lewis. An Experimental Comparison of Three Methods for Collision Handling in Virtual Environments. Human Factors and Ergonomics Society Annual Meeting Proceedings, 41:1273- 1277(5), 1997.
[16] A. Jacoff, E. Messina, B.A. Weiss, S. Tadokoro, Y. Nakagawa. Test Arenas and Performance Metrics for Urban Search and Rescue. Proc. of IEEE/RSJ In'l. Conf. on Intelligent Robots and Systems (IROS), volume 3, pages 3396-3403, December 2003.

[17] C.A. Johnson, J.A. Adams, K. Kawamura. Evaluation of an Enhanced Human-Robot Interface. Proc. of the IEEE Int'l Conf. on Systems, Man, and Cybernetics (SMC), pages 900-905, October 2003.

[18] R.W. Lindeman. Virtual Contact: The Continuum from Purely Visual to Purely Physical. Proc. of the 47th Annual Meeting of the Human Factors and Ergonomics Society (HFES), pages 2103-2107, October 2003.

[19] R.W. Lindeman, J.L. Sibert, E. Mendez-Mendez, S. Patil, D. Phifer. Effectiveness of Directional Vibrotactile Cuing on a BuildingClearing Task. Proc. of ACM CHI, pages 271-280, April 2005.

[20] R.W. Lindeman, J.R. Cutler. Controller Design for a Wearable, Near-Field Haptic Display. Proc. of the 11th Symp. on Haptic Interfaces for Virtual Environment and Teleoperator Systems, pages 397-403, March 2003.

[21] R.W. Lindeman, Y. Yanagida, H. Noma, K. Hosaka. Wearable Vibrotactile Systems for Virtual Contact and Information Display. Special Issue on Haptic Interfaces and Applications, Virtual Reality, volume 9, numbers 2-3, pages 203-213, 2006.

[22] F. Michaud, P. Boissy, H. Corriveau, A. Grant, M. Lauria, D. Labonté, R. Cloutier, M.-A. Roux, M.-P., Royer, D. Iannuzzi. Telepresence robot for home care assistance. Proc. American Association for Artificial Intelligence Spring Symp. on Multidisciplinary Collaboration for Socially Assistive Robotics, March 2006.

[23] R.R. Murphy, E. Rogers. Cooperative assistance for remote robot supervision. Presence, volume 5, number 2, pages 224-240, October 1995.

[24] C.W. Nielsen, M.A. Goodrich. Comparing the Usefulness of Video and Map Information in Navigation Tasks. Proc. of the ACM Conf. on Human-Robot Interaction, pages 95-101, March 2006.

[25] C.W. Nielsen, M.A. Goodrich, B. Ricks. Ecological interfaces for improving mobile robot teleoperation. IEEE Transactions on Robotics, volume 23, number 5, pages 927-941, October 2007.

[26] J. Ryu and G. J. Kim. Using a vibro-tactile display for enhanced collision perception and presence. Proc. of the ACM Symp. on Virtual Reality Software and Technology (VRST), pages 89-96, 2004.

[27] J. Scholtz. Evaluation methods for human-system performance of intelligent systems. Proc. of the 2002 Performance Metrics for Intelligent Systems (PerMIS) Workshop, 2002.

[28] A. Steinfeld, T. Fong, D. Kaber, M. Lewis, J. Scholtz, A. Schultz, M. Goodrich. Common Metrics for Human-Robot Interaction. Proc. of the 1st ACM SIGCHI/SIGART Conf. on Human-Robot Interaction, pages 33-40, March 2006.

[29] H.A. Yanco, J.L. Drury, J. Scholtz. Beyond Usability Evaluation: Analysis of Human-Robot Interaction at a Major Robotics Competition Human-Computer Interaction, volume 19, numbers 1-2, pages 117-149, June 2004.

[30] H.A. Yanco, M. Baker, R. Casey, B. Keyes, P. Thoren, J.L. Drury, D. Few, C. Nielsen, D. Bruemmer. Analysis of Human-Robot Interaction for Urban Search and Rescue. Proc. of the IEEE Symp. on Safety, Security and Rescue Robots, August 2006.

[31] J.S. Zelek, D. Asmar. A Robot's Spatial Perception Communicated via Human Touch. Proc. of the IEEE Int'l Conf. on Systems, Man, and Cybernetics (SMC), pages 454-461, October 2003. 